\title{
Measuring the acceleration of a rigid body
}

\author{
Peter G. Martin $^{\mathrm{a}, *}$, Gregory W. Hall ${ }^{\mathrm{b}}$, \\ Jeff R. Crandall ${ }^{\mathrm{a}}$ and Walter D. Pilkey ${ }^{\mathrm{a}}$ \\ a Department of Mechanical, Aerospace, and Nuclear \\ Engineering, Automobile Safety Laboratory, \\ University of Virginia, 1011 Linden Avenue, \\ Charlottesville, VA 22902, USA \\ ${ }^{\mathrm{b}}$ Exponent Failure Analysis Associates, 149 \\ Commonwealth Drive, Menlo Park, CA 94025, USA
}

Received 26 September 1997

Revised 10 July 1998

Two methods to measure the six-degree-of-freedom acceleration of a point on a rigid body are presented. The first, referred to as the periphery scheme, makes use of three clusters of accelerometers mounted orthogonal to each other and coincident with the axes of the point. One of the clusters consists of the three accelerometers attached to a cube-shaped triaxial angular rate sensor (ARS). The second method, called the compact cube scheme, uses a single 3accelerometer/ARS cluster that may be mounted anywhere on the rigid body. During impact tests with an instrumented rigid body, both methods produced measurements that were highly correlated near the time of peak acceleration. Whereas the compact cube scheme was more economical and easier to implement, the periphery scheme produced results that were less disrupted by instrument signal errors and noisy environments.

Keywords: Rigid body, rate sensor, accelerometer, acceleration, angular acceleration, impact, error model

\section{Introduction}

In biomechanical research with cadavers and anthropomorphic test dummies, head acceleration must be measured accurately to determine brain injury risk during motor vehicle safety tests. Acceleration at the center of the head determines the Head Injury Criteria (HIC) value, an established indicator of brain injury potential [7]. Angular head acceleration is also used as

\footnotetext{
*Corresponding author: Peter G. Martin, Automobile Safety Laboratory, 1011 Linden Avenue, Charlottesville, VA 22902, USA. E-mail:pgm5h@virginia.edu.
}

an injury criterion. This has prompted several efforts to accurately measure the six-degree-of-freedom acceleration of a rigid body.

If the linear acceleration of a point on a rigid body is desired, it might possibly be measured by simply mounting three orthogonal linear accelerometers near the point. But if the point is internal or if it is directly contacted during an impact, then sensors cannot be mounted nearby. The acceleration of such an inaccessible point can still be ascertained by measuring the acceleration of a second point on the rigid body (a reference point at any convenient location), along with the angular velocity and angular acceleration of the rigid body.

Measuring the angular acceleration of a rigid body can be particularly challenging because it requires several sensors. Arrays of linear accelerometers have proven to be a very reliable means of measuring the six-degree-of-freedom acceleration of a rigid body. Several schemes have been developed previously, including arrays of 15 accelerometers, nine accelerometers, and six accelerometers [5,9]. A nine-accelerometer array arranged in a 3-2-2-2 pattern has proven to be the one of the most reliable methods and has widespread use in impact biomechanics [6]. The following presentation compares the 3-2-2-2 array with new measurement schemes that combine accelerometers with sensors that measure angular velocity. The advantages and drawbacks of each measurement scheme are analyzed, and the appropriate applications of each are discussed.

\section{Theory: rigid body acceleration}

The acceleration vector of a fixed reference point on a rigid body, moving within an inertial frame, consists of the acceleration of the origin of the body-fixed reference frame and the tangential and centripetal accelerations at the reference point arising from rotation of the rigid body. Since the reference point is fixed to the rigid body, no Coriolis terms are present. In equation form, the acceleration of the reference point is expressed as:

$$
\vec{A}_{P}=\vec{A}_{0}+\vec{\omega} \times\left(\vec{\omega} \times \vec{r}_{P}\right)+\overrightarrow{\dot{\omega}} \times \vec{r}_{P}
$$


where: $\vec{A}_{P}$ - acceleration of the reference point, $P$, on the rigid body with respect to the inertial frame; $\overrightarrow{A_{0}}$ - acceleration of the origin, 0 , of the body-fixed reference frame with respect to the inertial frame; $\vec{\omega}-$ angular velocity of the rigid body frame; $\overrightarrow{\dot{\omega}}-$ angular acceleration of the rigid body frame; $\vec{r}_{P}$ - position of point, $P$, with respect to the origin, 0 , of the body-fixed frame.

The sensors that are needed to measure the acceleration of an inaccessible point (point 0 ) on (or within) the rigid body become evident when Eq. (1) is separated into $x, y$, and $z$ components:

$$
\begin{aligned}
A_{0 X}= & A_{P X}+r_{X}\left(\omega_{Y}^{2}+\omega_{Z}^{2}\right)-r_{Y}\left(\omega_{X} \omega_{Y}-\dot{\omega}_{Z}\right) \\
& -r_{Z}\left(\omega_{X} \omega_{Z}-\dot{\omega}_{Y}\right), \\
A_{0 Y}= & A_{P Y}-r_{X}\left(\omega_{X} \omega_{Y}+\dot{\omega}_{Z}\right)+r_{Y}\left(\omega_{X}^{2}+\omega_{Z}^{2}\right) \\
& -r_{Z}\left(\omega_{Y} \omega_{Z}-\dot{\omega}_{X}\right), \\
A_{0 Z}= & A_{P Z}-r_{X}\left(\omega_{X} \omega_{Z}-\dot{\omega}_{Y}\right)-r_{Y}\left(\omega_{Y} \omega_{Z}+\dot{\omega}_{X}\right) \\
& +r_{Z}\left(\omega_{X}^{2}+\omega_{Y}^{2}\right) .
\end{aligned}
$$

The vector $\vec{r}$ extends from point 0 to a point on the rigid body where acceleration can be measured (point $P$ ) using a triaxial arrangement of closely spaced accelerometers. The vector $\vec{r}$ is not usually difficult to determine, but the angular rate terms can present problems. A presentation of methods to solve Eqs (2)-(4) follows.

\section{The nine-accelerometer package}

The nine-accelerometer package (NAP), commonly referred to as the 3-2-2-2 array, consists of a set of triaxial accelerometers amid three sets of tangentially positioned biaxial accelerometer pairs. The geometry of the NAP is shown in Fig. 1. Under this layout, Padgaonkar demonstrated how the angular velocity terms in Eqs (2)-(4) are eliminated so that the angular acceleration can be determined directly at each point in time [5]. The equations for angular acceleration are:

$$
\begin{aligned}
& \dot{\omega}_{X}=\frac{\left(A_{1 Z}-A_{P Z}\right)}{2 \rho_{X}}-\frac{\left(A_{3 Y}-A_{P Y}\right)}{2 \rho_{Z}}, \\
& \dot{\omega}_{Y}=\frac{\left(A_{3 X}-A_{P X}\right)}{2 \rho_{Z}}-\frac{\left(A_{2 Z}-A_{P Z}\right)}{2 \rho_{Y}}, \\
& \dot{\omega}_{Z}=\frac{\left(A_{2 Y}-A_{P Y}\right)}{2 \rho_{Y}}-\frac{\left(A_{1 X}-A_{P X}\right)}{2 \rho_{X}} .
\end{aligned}
$$

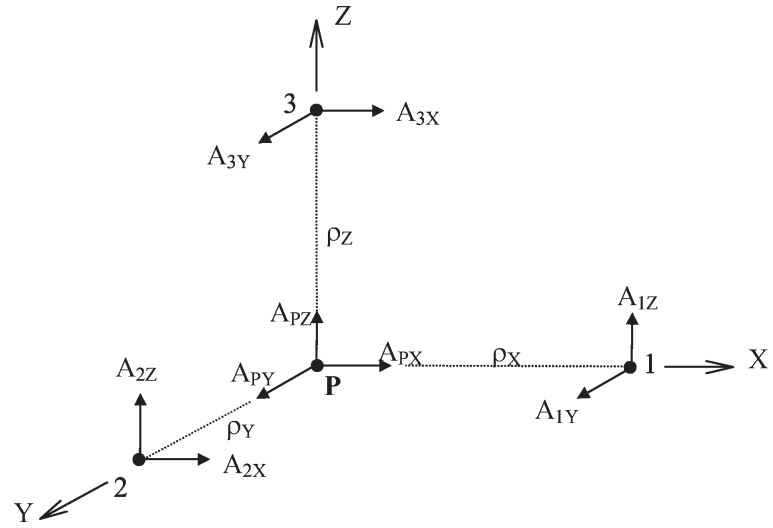

Fig. 1. The nine-accelerometer package.

Unlike the ideal planar arrangement shown in Fig. 1, two (or three) accelerometers cannot occupy the same point in space. DiMasi developed an algorithm to compensate for cross-products of angular velocity and centripetal accelerations arising from the non-coplanar geometry [1].

The NAP was developed specifically for anthropomorphic test dummies used in automobile safety assessments. The NAP fits inside the dummy head and works best when the accelerometers are affixed directly to the inner surface of a hollow head form having machined inner mounting surfaces. However, a mounting fixture is available so that the NAP may be placed inside an unmachined head form [6].

Conceivably, a mounting fixture could also be used to affix the NAP to the surface of any rigid body so that it could be used to measure the linear acceleration of an inaccessible point. Equations (2)-(4) could then be solved after defining the vector, $\vec{r}$, from the inaccessible point to the origin of the NAP array and finding the angular velocity terms by integrating the angular acceleration quantities determined via Eqs (5)-(7).

There are difficulties with using the NAP in this manner for impact tests, however. The non-coplanar layout of the array will require a strong fixture to assure that the unit does not deform or resonate relative to the rigid body upon impact. Depending on the size of the rigid body, the addition of such a fixture could substantially change its inertial properties. Typically, a NAP mounting fixture has a mass of about $200 \mathrm{~g}$, and the three 2-accelerometer clusters are spaced about $5 \mathrm{~cm}$ away from the center triax. Any effort to reduce the size of the array will magnify the effects of signal noise. For example, if $\rho_{X}$ in Eq. (5) is small, then the signals produced by $A_{1 Z}$ and $A_{P Z}$ will be nearly identical and the difference between them will be due 
mostly to noise. Thus the accuracy of $\dot{\omega}_{X}$, calculated from $A_{1 Z}$ and $A_{P Z}$, will be degraded.

\section{New methods using angular rate sensors}

The acceleration of an inaccessible point on or within a rigid body and the angular acceleration of the rigid body may obtained using measurement schemes consisting of accelerometers and a three-axis angular rate sensor (ARS cube). Two such schemes are presented.

\subsection{Peripheral scheme}

The layout of this scheme is shown in Fig. 2. Three sets of accelerometer clusters are mounted to the surface of the rigid body, but spaced apart so that each of the three orthogonal axes originating from the inaccessible point coincides with the sensitive axis of a triax. Hence, the accelerometers are positioned peripherally around the rigid body. In addition, an ARS cube measures the angular velocity vector. The ARS cube also serves as a mount for one of the three accelerometer clusters. This arrangement is shown to occur at point 3 in Fig. 2. Thus, sensors are mounted at three places on the rigid body.

Under this scheme, the centripetal acceleration and the linear acceleration along the coordinate axes at the peripheral points (points 1, 2, and 3 in Fig. 2) are the only measurements needed to determine the linear acceleration of the inaccessible point (point 0 in Fig. 2). Thus, only six sensors are required: three accelerometers (the boldfaced vectors in Fig. 2) and the three sensors within the ARS cube. As such, Eqs (2)-(4) are re-

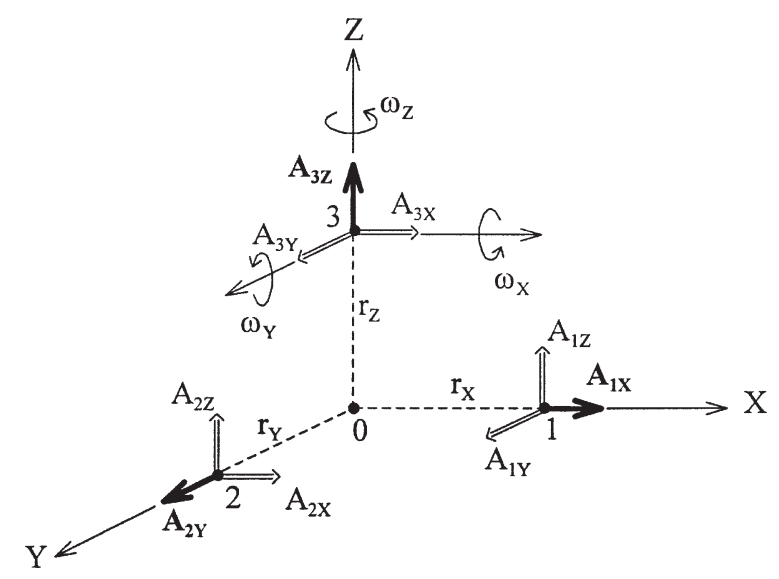

Fig. 2. Peripheral sensor mounting scheme. duced so that acceleration at the inaccessible point may be found from Eqs (8)-(10).

$$
\begin{aligned}
& A_{0 X}=A_{1 X}+r_{X}\left(\omega_{Y}^{2}+\omega_{Z}^{2}\right), \\
& A_{0 Y}=A_{2 Y}+r_{Y}\left(\omega_{X}^{2}+\omega_{Z}^{2}\right), \\
& A_{0 Z}=A_{3 Z}+r_{Z}\left(\omega_{X}^{2}+\omega_{Y}^{2}\right) .
\end{aligned}
$$

Computation of angular acceleration makes use of the six additional accelerometers (shown as doublelined vectors in Fig. 2). Only three of these extra accelerometers are actually needed to compute the angular acceleration components. For example, if only accelerometers $A_{1 Y}, A_{3 X}$, and $A_{3 Y}$ were retained, the angular acceleration terms could be computed from Eqs (11)-(13).

$\dot{\omega}_{X}=\frac{\left(A_{2 Y}-A_{3 Y}\right)}{r_{Z}}+\omega_{Y} \omega_{Z}+\frac{r_{Y}\left(\omega_{X}^{2}+\omega_{Z}^{2}\right)}{r_{Z}}$,
$\dot{\omega}_{Y}=\frac{\left(A_{3 X}-A_{1 X}\right)}{r_{X}}+\omega_{X} \omega_{Z}+\frac{r_{Z}\left(\omega_{X}^{2}+\omega_{Y}^{2}\right)}{r_{X}}$,
$\dot{\omega}_{Z}=\frac{\left(A_{1 Y}-A_{3 Y}\right)}{r_{X}}-\omega_{X} \omega_{Y}-\frac{r_{Y}\left(\omega_{X}^{2}+\omega_{Z}^{2}\right)}{r_{X}}$.

With the three extra accelerometers, each component of angular acceleration may be computed using two different sets of accelerometers. A least-square fit between the two independent results provides a more reliable computation, while the redundancy guards against lost data due to the failure of a single data channel.

Also, if all nine accelerometers are used, it will not be necessary to maintain the parallel alignment of triax clusters shown in Fig. 2. This is advantageous for oddshaped rigid bodies upon which an orthogonal mounting system is not possible. As long as the body-fixed axes pass through the center of the triaxes and the orientation of each triax is known with respect to the body-fixed frame, the acceleration and velocity vectors in Eqs (8)-(10) may be resolved.

The peripheral layout of the sensors has several advantages over other six-degree-of-freedom measurement schemes cited earlier, including the NAP. The periphery scheme - as opposed to the NAP - does not require angular acceleration terms to compute the linear acceleration at the inaccessible point. Since the differences between pairs of accelerometers are used to determine angular acceleration, the NAP scheme is open to errors due to signal noise and mismatch between the response vectors of the accelerometer pairs. 
Whereas the NAP scheme computes angular velocity by integrating angular acceleration, the periphery scheme measures angular velocity directly. As such, the periphery scheme is not susceptible to error accumulation inherent in numerical integration. Furthermore, the peripheral layout maximizes the distance between accelerometer triaxes, which minimizes the effects of signal noise and the sensor mismatch on the computation of angular acceleration. Also, by distributing the sensors around the surface of the rigid body on three relatively small mounting plates, the periphery layout has a limited effect on the rigid body's center of gravity location.

The primary disadvantage of the periphery scheme is that a minimum of three mounting locations are required. Measuring the distance from the inaccessible point to each accelerometer may be a challenge without sophisticated positioning equipment, and it may be difficult to attain sensor orthogonality. Also, since the sensors are distributed around the surface of the rigid body, there is a higher risk of contact between the sensors and the experimental apparatus during testing which could lead to sensor damage.

\subsection{Compact cube scheme}

This scheme is shown in Fig. 3, where three linear accelerometers are attached to the ARS cube and the cube is then mounted to the surface of the rigid body. This scheme greatly simplifies mounting procedures, but must use numerically differentiated ARS output to compute acceleration.

Unlike the periphery layout, the axes of the accelerometers in the compact cube scheme of Fig. 3 are not coincident with the axes of the inaccessible point, point 0 . Thus, the angular acceleration terms cannot be dropped from Eqs (2)-(4) in the computation of acceleration at the inaccessible point. However, Eqs (2)-(4) may be simplified by aligning one of the local ARS

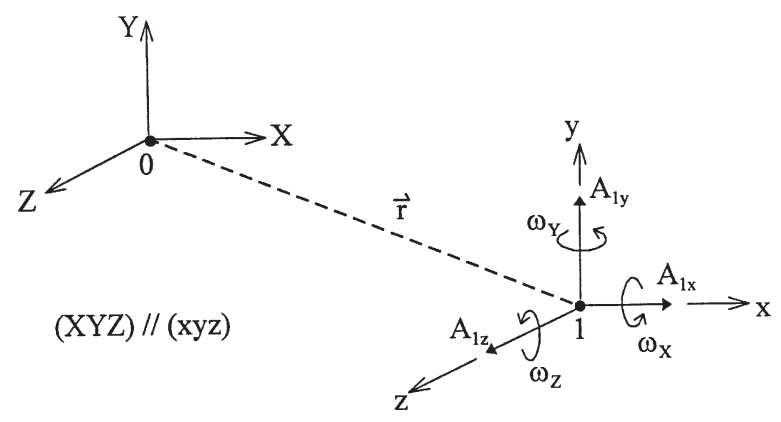

Fig. 3. Compact cube scheme. (xyz) axes with one of the body-fixed (XYZ) axes. For example, if $(\mathrm{x}, \mathrm{X})$ are coincident and $(\mathrm{y}, \mathrm{Y})$ and $(\mathrm{z}, \mathrm{Z})$ are parallel, then $r_{Y}$ and $r_{Z}$ are zero and Eqs (2)-(4) reduce to:

$$
\begin{aligned}
& A_{0 X}=A_{1 X}+r_{X}\left(\omega_{Y}^{2}+\omega_{Z}^{2}\right), \\
& A_{0 Y}=A_{1 Y}-r_{X}\left(\omega_{X} \omega_{Y}+\dot{\omega}_{Z}\right), \\
& A_{0 Z}=A_{1 Z}-r_{X}\left(\omega_{X} \omega_{Z}-\dot{\omega}_{Y}\right) .
\end{aligned}
$$

Alternatively, the ARS cube may be placed anywhere on the rigid body in any orientation by defining a third coordinate system (ijk) originating at the center of the ARS cube with one of its axes passing through point 0 . First, the acceleration and angular rate vectors are transformed from their local (xyz) frame to the (ijk) frame. Then acceleration at point 0 is computed in the (ijk) frame using equations similar to Eqs (14)-(16). Finally, results are transformed to the (XYZ) frame.

In the compact cube scheme, the angular acceleration terms, $\dot{\omega}_{X}, \dot{\omega}_{Y}$, and $\dot{\omega}_{Z}$ are determined by numerically differentiating the corresponding angular velocity quantities. (A three-point centered difference formula was chosen for the analysis that follows.) This is the main drawback of this scheme: numerical differentiation is always a noise amplifying process, especially if the initial data is somewhat noisy. The results of the differentiation require the subsequent application of a low-pass filter to remove noise. However, this will also remove any high-frequency components of angular acceleration that actually exist.

The effects of numerical differentiation may be tempered by selecting the ARS mounting site judiciously. For example, if rotation is expected to occur mostly about the rigid body's X-axis, then the ARS may be mounted such that its local $\mathrm{X}$-axis coincides with bodyfixed $\mathrm{X}$-axis. The angular rate terms and the error associated with them will have a minimal effect on the acceleration of the inaccessible point (point 0) as computed by Eqs (14)-(16).

The appeal of the compact cube arrangement is its compactness and its ease of mounting. The small cube/accelerometer arrangement limits its influence on the inertial properties of the rigid body. Meantime, the cube-shaped ARS serves as an orthogonal mounting block for the accelerometers and can usually be attached to the rigid body with ease. Moreover, only three sensor orientation measurements are needed to transform sensor readings from their local (xyz) frame to the body-fixed (XYZ) frame. As such, the cube may be mounted at a convenient location that is computationally advantageous and is least likely to interfere with surrounding equipment. 


\section{Co-planar scheme (not evaluated)}

The attraction of the periphery layout is that the computations of acceleration at the inaccessible point do not require angular acceleration terms, thus reducing error. However, it is not always possible to align the accelerometers with the axes of an inaccessible point. Although it was not evaluated as such, the periphery scheme may be laid out in a co-planar configuration as shown in Fig. 4. This layout allows the sensors to be arranged on a flat mounting plate that may be fastened to the rigid body at any site. The angular acceleration is computed by Eqs (17)-(19). The linear acceleration of an inaccessible point (point 0 in Fig. 4 ) is then obtained by measuring the vector $\vec{r}$ (point 0 to point 1 in Fig. 4), and applying Eqs (2)-(4):

$$
\begin{gathered}
\dot{\omega}_{X}=\frac{A_{1 Z}-A_{2 Z}}{\rho_{y}}-\omega_{Y} \omega_{Z}, \\
\dot{\omega}_{Y}=\frac{A_{1 X}-A_{3 X}}{\rho_{z}}+\omega_{X} \omega_{Z}, \\
\dot{\omega}_{Z}=\frac{A_{2 X}-A_{1 X}}{\rho_{y}}-\omega_{X} \omega_{Z} .
\end{gathered}
$$

Theoretically, Eqs (17)-(19) can be solved using only the data from the six accelerometers by employing a numerical stepwise integration procedure. However, this procedure is inherently unstable because angular velocity is computed cumulatively [4]. The slightest signal irregularity sets off a rapid growth of error in the angular acceleration calculations. This instability is overcome by adding an ARS cube which measures angular velocity directly.

The co-planar arrangement benefits from the same computational advantages over the NAP as does the periphery scheme, and a co-planar mounting fixture

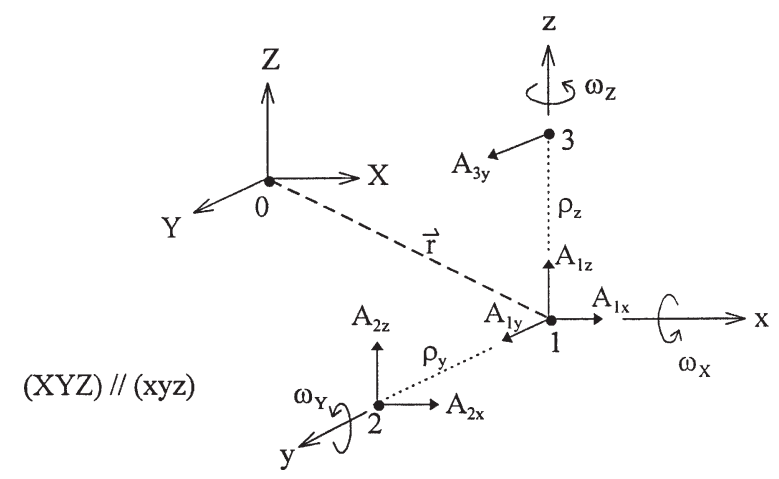

Fig. 4. Co-planar 6 accelerometer/ARS cube configuration. would be much simpler than the NAP's. Moreover, a 3-3-3/ARS co-planar scheme could be easily implemented by using triaxial accelerometers at all three locations (points 1, 2, and 3 in Fig. 4). This would enable each angular acceleration term to be computed using two different sets of accelerometers. Such redundancy provides extra data to improve the reliability of the results. However, like the NAP array, the minimum size of the co-planar array is limited due to problems associated with the noise-to-signal ratio.

\section{Comparison methods}

The various measurement schemes were compared using a specially machined Hybrid-II dummy head form with a mass of $3.7 \mathrm{~kg}$. Three uniaxial accelerometers (Model 7264A, Endevco, San Juan Capistrano, CA), each having a mass of one gram, were mounted within the head form at its center of gravity (CG) and aligned in the $\mathrm{X}, \mathrm{Y}$ and $\mathrm{Z}$ directions as shown in Fig. 5. Additionally, two triaxial accelerometers (Endevco Model 7267A), each having a mass of $50 \mathrm{~g}$, were mounted to the side and top surfaces of the head form coincident with the Y- and Z-axes, respectively. A magneto-hydrodynamic ARS cube (Dynacube, Applied Technology Associates, Albequerque, NM) was used to measure angular velocity, and three more linear accelerometers (Endevco Model 7264A) were fastened to the orthogonal faces of the cube. The cube, measuring $34 \mathrm{~cm}^{3}$ and having a mass of $80 \mathrm{~g}$, was mounted to the posterior skull cap so that the sensitive axis of an accelerometer (A11 in Fig. 5) was coincident with the $\mathrm{X}$-axis. Table 1 shows which sensors were employed by the various measurement schemes.

Impact tests with the instrumented head form were carried out using a vertical drop tower. The head form, minus the vinyl skin covering, was affixed to a flexible dummy neck. The neck was connected to a triangular aluminum support bracket via a clevis. The clevis allowed the rotational position of the head to be adjusted about two axes. The bracket was connected to a drop tower by means of two linear bearings.

Drop tests were performed under two impact conditions: direct contact as shown in Fig. 6 and non-contact as shown in Fig. 7. The direct contact test was characterized by a brief period of very high linear and angular accelerations. This was meant to simulate the impact of a motorist's head with a dash panel. Most of the motion was in the $\mathrm{X}-\mathrm{Z}$ plane so that the rotation 

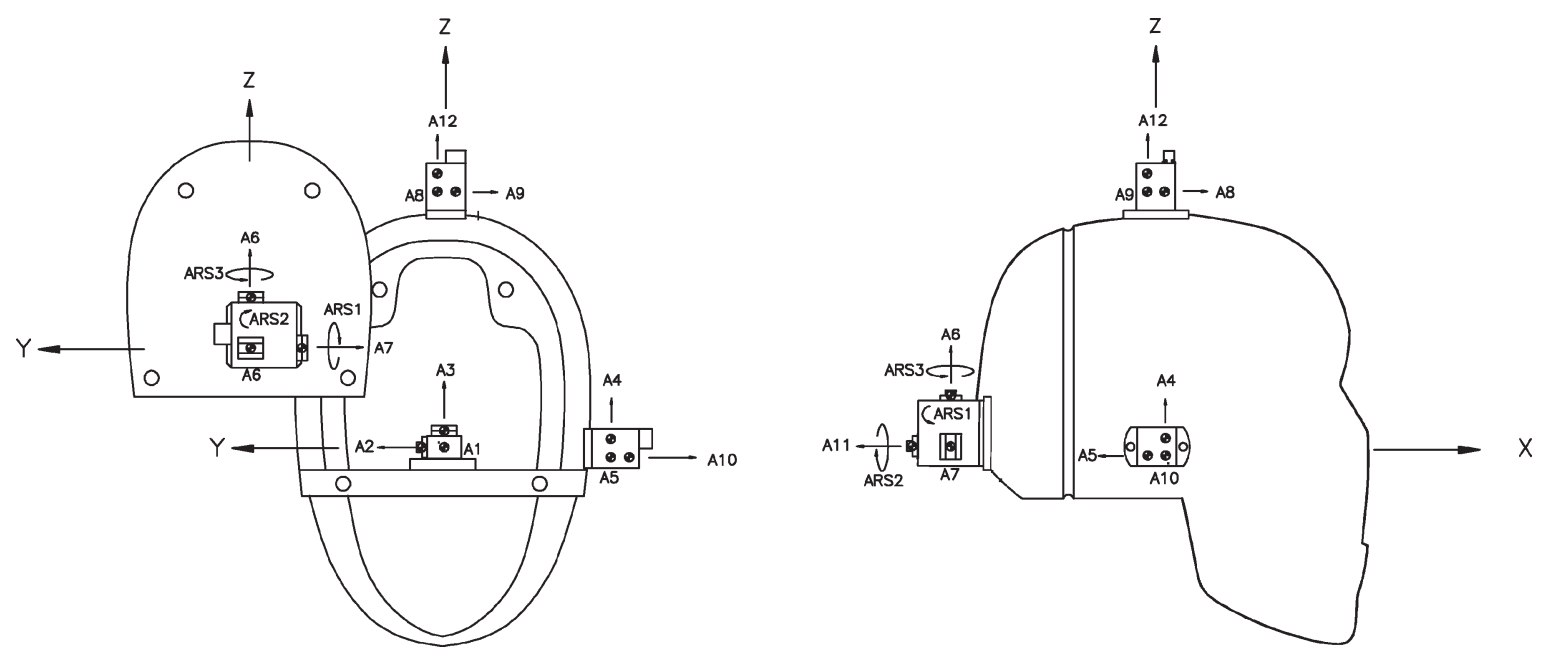

Fig. 5. Sensor positions in specially machined head form. On left, rear view shows skull cap partially removed to reveal accelerometer triax at the CG.

Table 1

Sensors employed by each measurement scheme

\begin{tabular}{|c|c|c|c|c|c|c|}
\hline \multirow[t]{2}{*}{ Scheme } & \multicolumn{3}{|c|}{ Linear acceleration at $\mathrm{CG}$} & \multicolumn{3}{|c|}{ Rigid body angular acceleration } \\
\hline & Accelerometers required & ARS? & $\begin{array}{c}\text { Number of } \\
\text { sensors }\end{array}$ & Accelerometers required & $\overline{\text { ARS? }}$ & $\begin{array}{c}\text { Number of } \\
\text { sensors }\end{array}$ \\
\hline NAP & Not used to find CG accel. & - & - & $\begin{array}{l}\text { A1x, A2y, A3z, A4x, A5z, } \\
\text { A6y, A7z, A8x, A9y }\end{array}$ & No & 9 \\
\hline Peripheral & $\mathrm{A} 10 \mathrm{y}, \mathrm{A} 11 \mathrm{x}, \mathrm{A} 12 \mathrm{z}$ & Yes & 6 & $\mathrm{~A} 7 \mathrm{z}, \mathrm{A} 8 \mathrm{x}, \mathrm{A} 9 \mathrm{y}, \mathrm{A} 10 \mathrm{y}, \mathrm{A} 11 \mathrm{x}$ & Yes & 8 \\
\hline Comp. Cube & A10x, A6y, A7z & Yes & 6 & None & Yes & 3 \\
\hline Direct CG & $\mathrm{A} 1 \mathrm{x}, \mathrm{A} 2 \mathrm{y}, \mathrm{A} 3 \mathrm{z}$ & No & 3 & Not Applicable & - & - \\
\hline
\end{tabular}

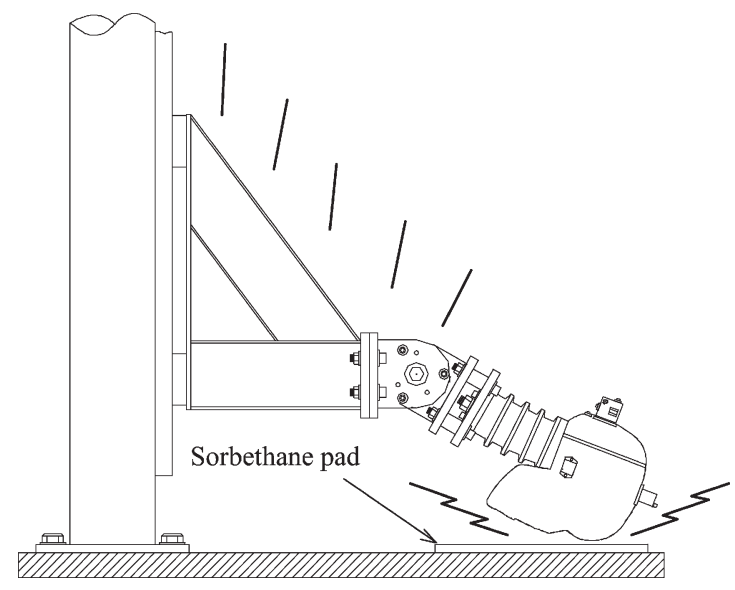

Fig. 6. Direct contact, $30^{\circ}$ pitch, $0^{\circ}$ roll.

of the head form could be tracked with a high-speed video camera.

The non-contact test produced lower accelerations over a prolonged period. This test was meant to simulate head motion commonly experienced by belted mo-

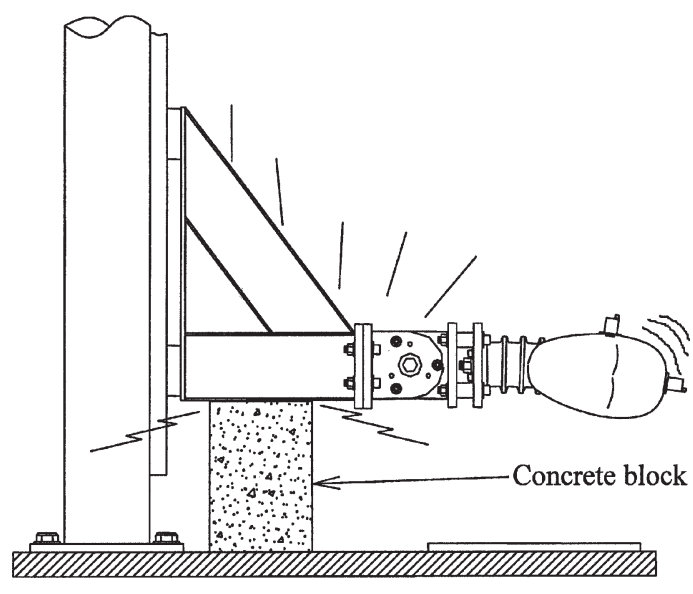

Fig. 7. Non-contact, $0^{\circ}$ pitch, $90^{\circ}$ roll.

torists in a crash. Unlike the contact test, significant rotation occurred about all three axes. In both tests, the drop height was $1.2 \mathrm{~m}$ and the impact speed was $4.8 \mathrm{~m} / \mathrm{s}$. Time histories covering roughly $50 \mathrm{~ms}$ were analyzed for both tests. 
Electronic data for both tests were collected with a DSP Technology Transient Acquisition and Processing System, Model TRAQ P. The sampling frequency was set at $10 \mathrm{kHz}$ with a low-pass filter set at $1650 \mathrm{~Hz}$ corresponding to the impact instrumentation specifications set forth in SAE J211 [8]. The polarity of the sensors was reversed where needed so that a positive output corresponded with the positive (XYZ) axes of the head frame as defined in Fig. 5. High-speed video at 1000 frames per second was taken of the contact test only, since all motion was in a single plane.

\section{Results}

\subsection{Angular acceleration comparison}

Figures $8 \mathrm{a}-\mathrm{d}$ are overlays of the angular acceleration time histories produced by each scheme. Although a $1650 \mathrm{~Hz}$ filter was imposed on all sensors during data collection, the signals required subsequent filtering at a lower frequency. Figure 8 a shows the non-contact test time-histories without subsequent filtering. The compact cube data is particularly noisy due to the intrinsic problems with numerical differentiation. Moreover,

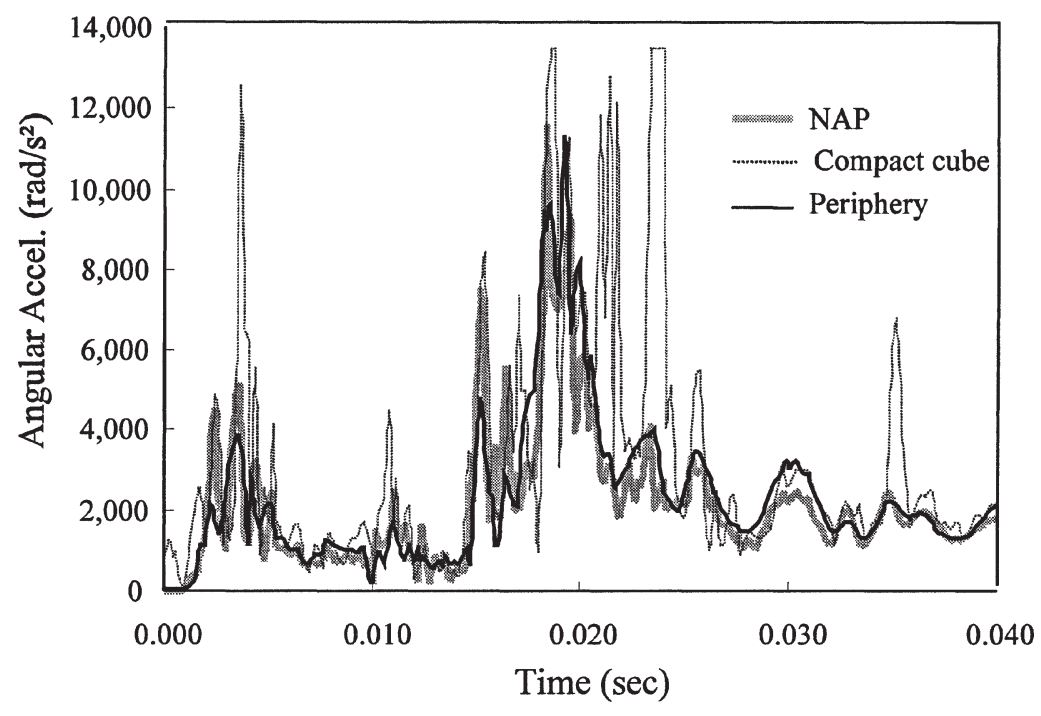

Fig. 8a. Non-contact test, unfiltered resultant acceleration.

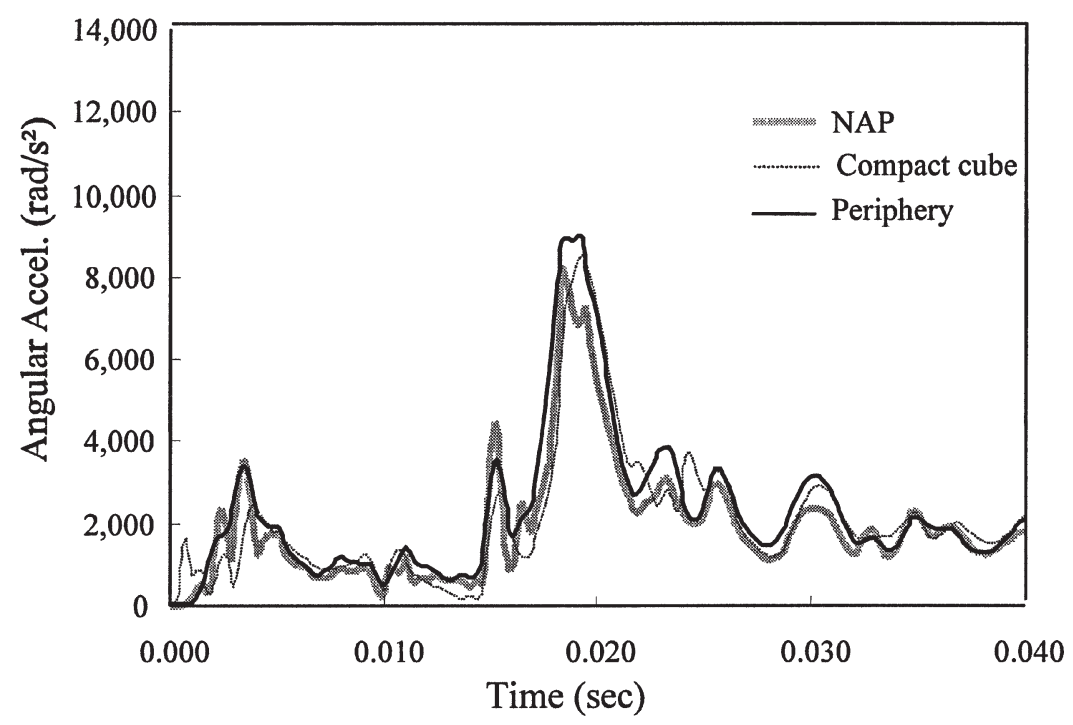

Fig. 8b. Non-contact test, filtered resultant acceleration. 


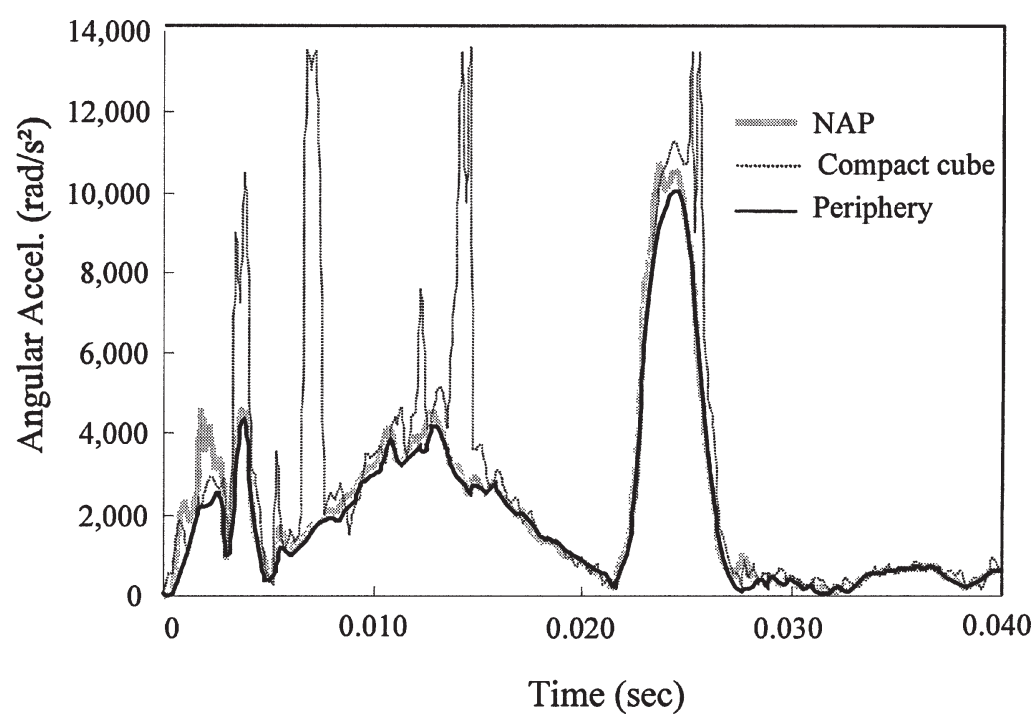

Fig. 8c. Contact test, unfiltered resultant acceleration.

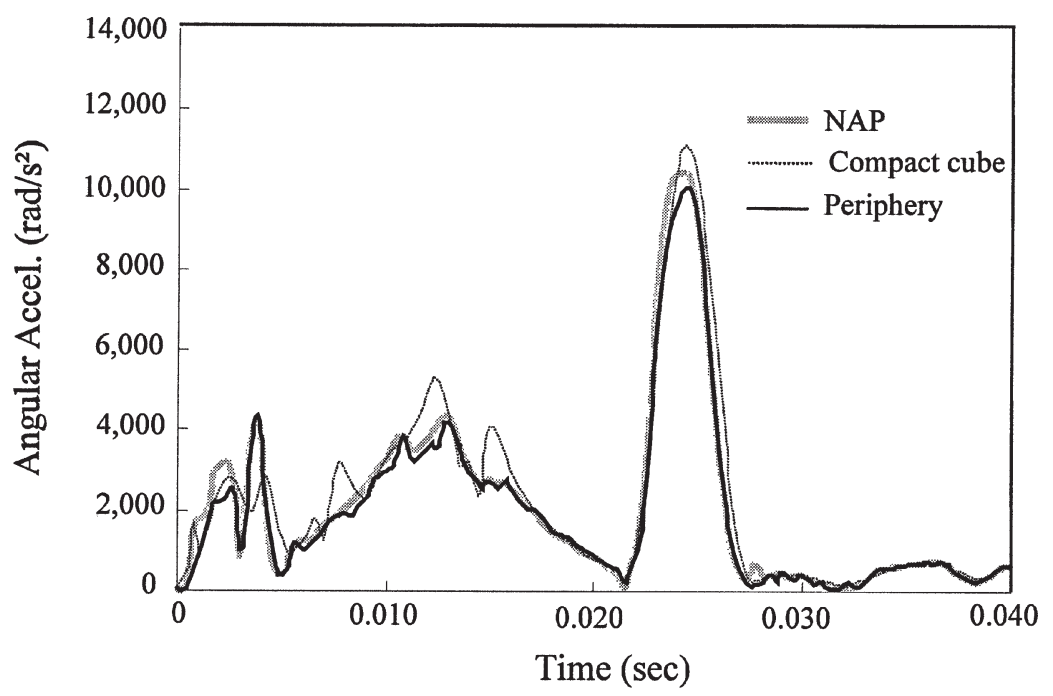

Fig. 8d. Contact test, filtered resultant acceleration.

the NAP time-history is noisier than that of the periphery scheme. The NAP scheme computes angular acceleration from the difference of two pairs of accelerometers, while the periphery scheme uses just one pair. The extra pair in the NAP scheme compounds the effects of noisy accelerometer outputs. Figure $8 \mathrm{~b}$ shows the improvement when the same data is digitally filtering using a 4-pole Butterworth filter with a $600 \mathrm{~Hz}$ cutoff frequency on accelerometer signals and a $300 \mathrm{~Hz}$ cut-off frequency on ARS signals.

In the contact test, the head struck a sorbethane pad that effectively damped out high frequency vibrations. The contact test overlays are shown in Fig. 8c with no post-test filtering and Fig. 8d with the filtered data. Filtering had very little effect on the NAP and periphery time-histories, but ARS data requires filtering even in the presence of a noise-free environment.

In addition to the overlays in Figs $8 \mathrm{a}-\mathrm{d}$, the angular acceleration time-histories in the contact test were compared analytically. A linear least-square analysis of the differences between a pair of curves measured the fit of the acceleration values to a straight-line gradient. The Pearson correlation coefficient associated with that fit provided an indirect measure of the agreement between the two schemes. The correlation coefficient was evaluated for the $0-2.5 \mathrm{~ms}$ interval between two 
curves and was assigned to the midpoint of the interval (time $t=1.3 \mathrm{~ms}$ ). The interval was then shifted ahead by one data point $(0.1 \mathrm{~ms})$ and the correlation coefficient was re-evaluated. This process was repeated for the entire time-history to produce the time-varying correlation coefficient curves in Fig. 9. Similar results were obtained for the filtered non-contact test data.

At lower acceleration values, the correlation coefficients drop well below one. However, near the peak value (which is of much greater interest), the correlation coefficients are very close to one indicating a high degree of fidelity among the three schemes. Thus, any differences among the acceleration time-histories near the peak values are mostly scaling effects due to sensor miscalibrations.

\section{Linear acceleration comparison}

The linear acceleration time-histories computed at the inaccessible point (the CG of the head) are shown in Fig. 10a for the non-contact test and Fig. 10b for the contact test. In both figures, the time-histories were produced with all accelerometer signals filtered at $600 \mathrm{~Hz}$ and ARS signals filtered at $300 \mathrm{~Hz}$. Table 2 lists the peak acceleration magnitudes produced in both tests by the various schemes with and without post-test filtering. The filters remove noise and vibrations from the data and bring the time-histories into closer agreement. Moreover, the contact test results show that the filters do not significantly alter time-histories in which vibrations are absent.

\section{Angular velocity and rotation}

The angular velocity comparison is shown in Figs 11a-b. The high frequency components seen in acceleration data do not appear in the angular velocity data. The ARS sensors are insensitive to linear vibrations [3], while the process of integrating NAP data effectively filters high frequency components. Much of the noise that does appears in the ARS time-histories is due to sensor malfunctions, such as the one occurring at time $t=0.014 \mathrm{~s}$ in the contact test. These anomalies appear in tests of all types including those in other impact studies that were not reported herein, and they translate into sharp spikes during numerical differentiation. Hence differentiated ARS data (collected with the sensor model cited earlier) will almost always require filtering near $300 \mathrm{~Hz}$.
Rotation during the contact test is shown in Fig. 12. The effects of integration are apparent as both the ARS and NAP data drift further from the high-speed video data as time marches on.

\section{Error analysis}

The precision required of the sensor position measurements when using the periphery and compact cube schemes may be assessed by examining error sensitivity in the calculations. In the general case, let the measure of acceleration, $A$, be a function of $n$ independent variables:

$$
A=f\left(a_{1}, a_{2}, \ldots, a_{n}\right)
$$

The $a$ are the parameters of the design equation and are in error by $\pm \Delta a_{1}, \pm \Delta a_{2}, \ldots, \pm \Delta a_{n}$, respectively. These errors will cause an error $\Delta A$ in the computed $A$ :

$$
\begin{aligned}
A+ & \Delta A \\
& =f\left(a_{1} \pm \Delta a_{1}, a_{2} \pm \Delta a_{2}, \ldots, a_{n} \pm \Delta a_{n}\right) .
\end{aligned}
$$

Taking a probabilistic approach and using the theory of random algebra and the algebra of expectation, it is standard practice to specify the $50 \%$ confidence as the most probable error. When the $\Delta a$ are considered not as absolute limits of error, but as probable errors, the proper method of combining such errors (neglecting second order terms) is according to the root-sum square formula [2]:

$$
\begin{aligned}
\Delta A= & \left(\left(\Delta a_{1} \frac{\partial f}{\partial a_{1}}\right)^{2}+\left(\Delta a_{2} \frac{\partial f}{\partial a_{2}}\right)^{2}\right. \\
& \left.+\cdots+\left(\Delta a_{n} \frac{\partial f}{\partial a_{n}}\right)^{2}\right)^{1 / 2}
\end{aligned}
$$

Error sensitivity analysis may be applied to the periphery and compact cube schemes by considering the precision of the sensor position measurements. Unless a high-precision positioning device is available, errors in the length measurements from the inaccessible point to the triaxes are conservatively estimated to be as much as $\pm 5 \%$ in each direction. Moreover, the maximal error in the orientation of the triaxes and the ARS cube may be $10^{\circ}$ from their respective axes, which would lead to perceived signal errors of $1-\cos \left(10^{\circ}\right)$, or $\pm 1.5 \%$. The error sensitivity in computing the $\mathrm{X}$-component of acceleration at the inaccessible point 


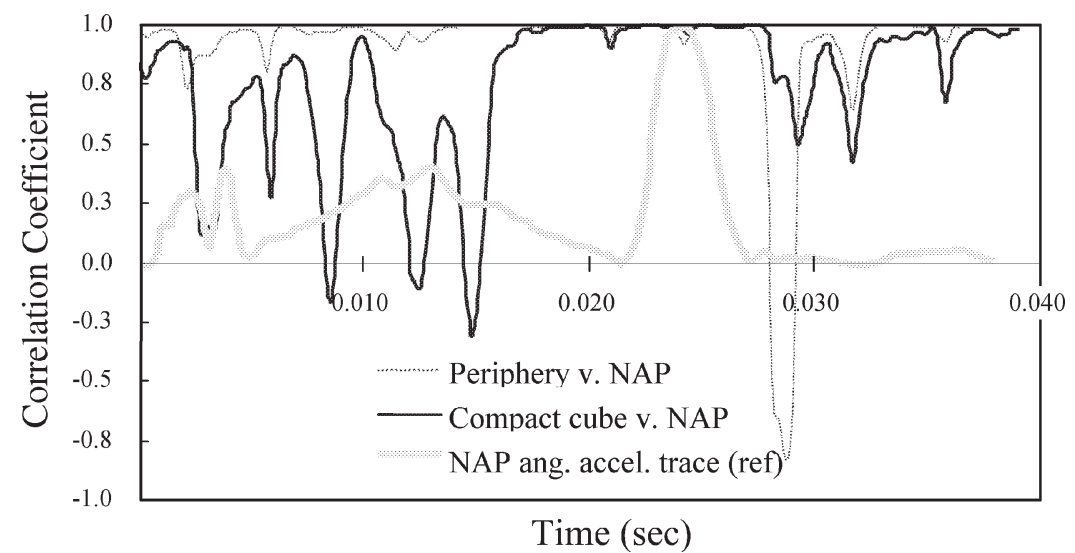

Fig. 9. Contact test, filtered angular acceleration correlation coefficient.

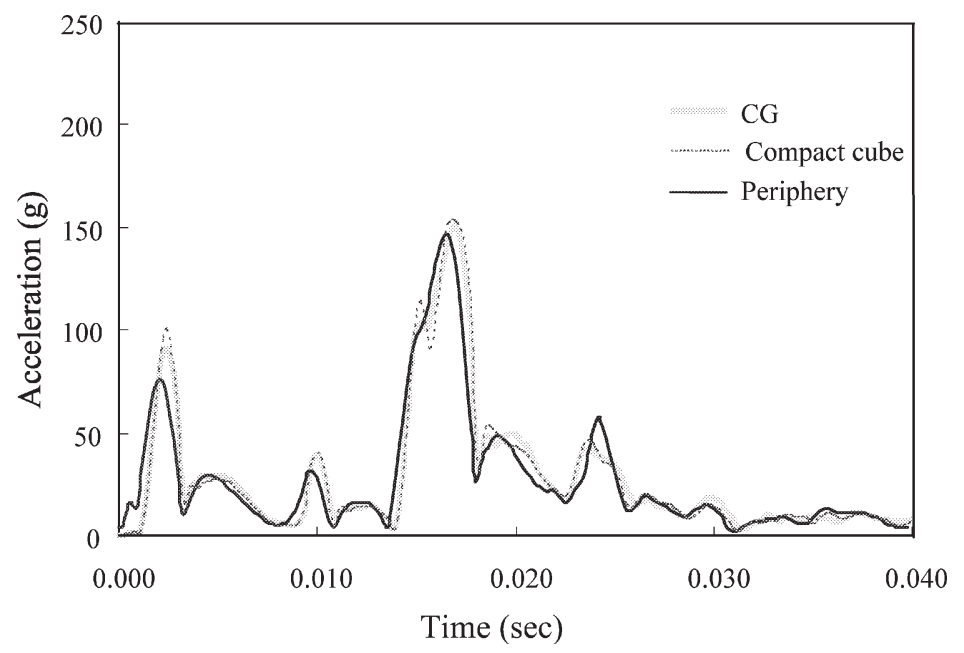

Fig. 10a. Non-contact test, filtered CG resultant acceleration.

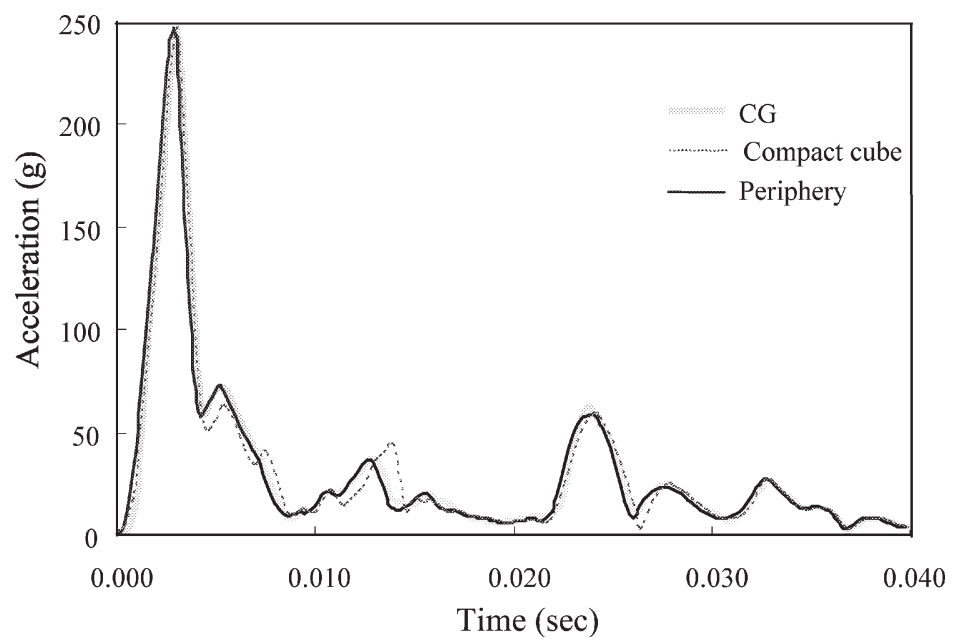

Fig. 10b. Contact test, filtered CG resultant acceleration. 
Table 2

Test results - peak magnitudes of acceleration with and without post-test filtering

\begin{tabular}{|c|c|c|c|c|c|c|c|c|}
\hline \multirow[t]{3}{*}{ Scheme } & \multicolumn{4}{|c|}{ Non-contact test (see Fig. 6) } & \multicolumn{4}{|c|}{ Contact test (see Fig. 5) } \\
\hline & \multicolumn{2}{|c|}{$\begin{array}{c}\text { Angular acceleration } \\
\left(\mathrm{rad} / \mathrm{s}^{2}\right)\end{array}$} & \multicolumn{2}{|c|}{$\begin{array}{l}\text { Linear acceleration } \\
\text { at the } \mathrm{CG}(\mathrm{g})\end{array}$} & \multicolumn{2}{|c|}{$\begin{array}{l}\text { Angular acceleration } \\
\qquad\left(\mathrm{rad} / \mathrm{s}^{2}\right)\end{array}$} & \multicolumn{2}{|c|}{$\begin{array}{l}\text { Linear acceleration } \\
\text { at the } \mathrm{CG}(\mathrm{g})\end{array}$} \\
\hline & Unfiltered & Filtered & Unfiltered & Filtered & Unfiltered & Filtered & Unfiltered & Filtered \\
\hline NAP/CG & 11,627 & 8179 & 159 & 151 & 10,772 & 10,447 & 249 & 246 \\
\hline Periphery & 11,344 & 9028 & 157 & 154 & 10,049 & 10,047 & 259 & 247 \\
\hline Comp. Cube & 31,077 & 10,570 & 190 & 168 & 35,939 & 11,100 & 266 & 254 \\
\hline
\end{tabular}

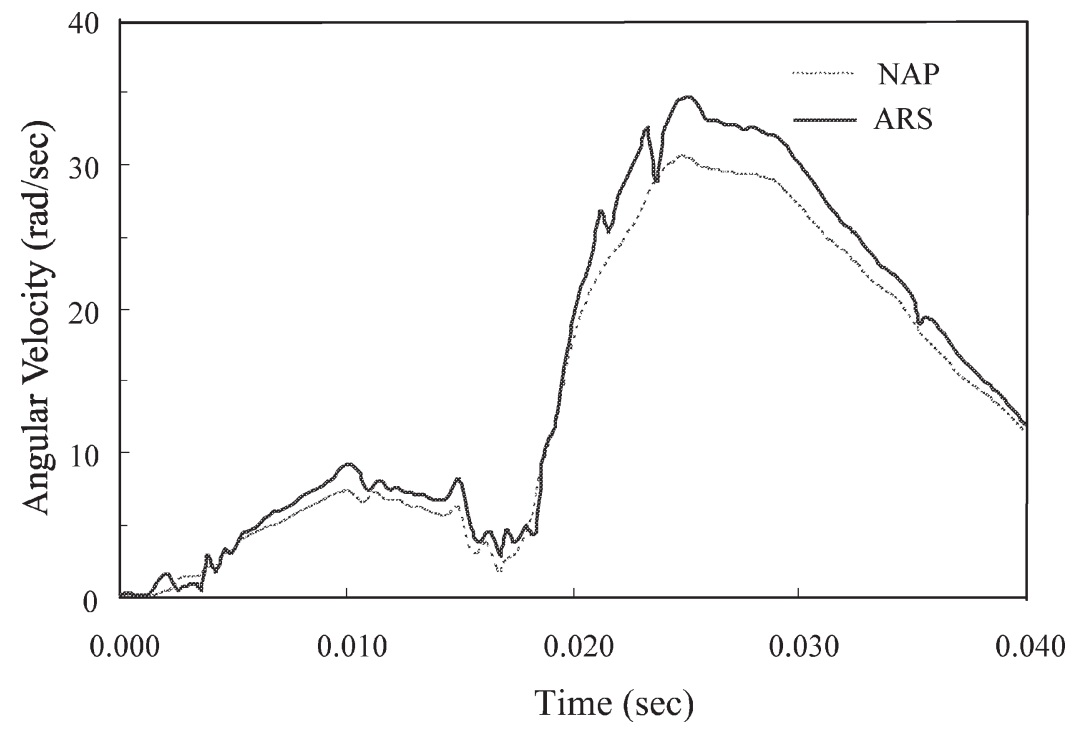

Fig. 11a. Non-contact test, resultant angular velocity.

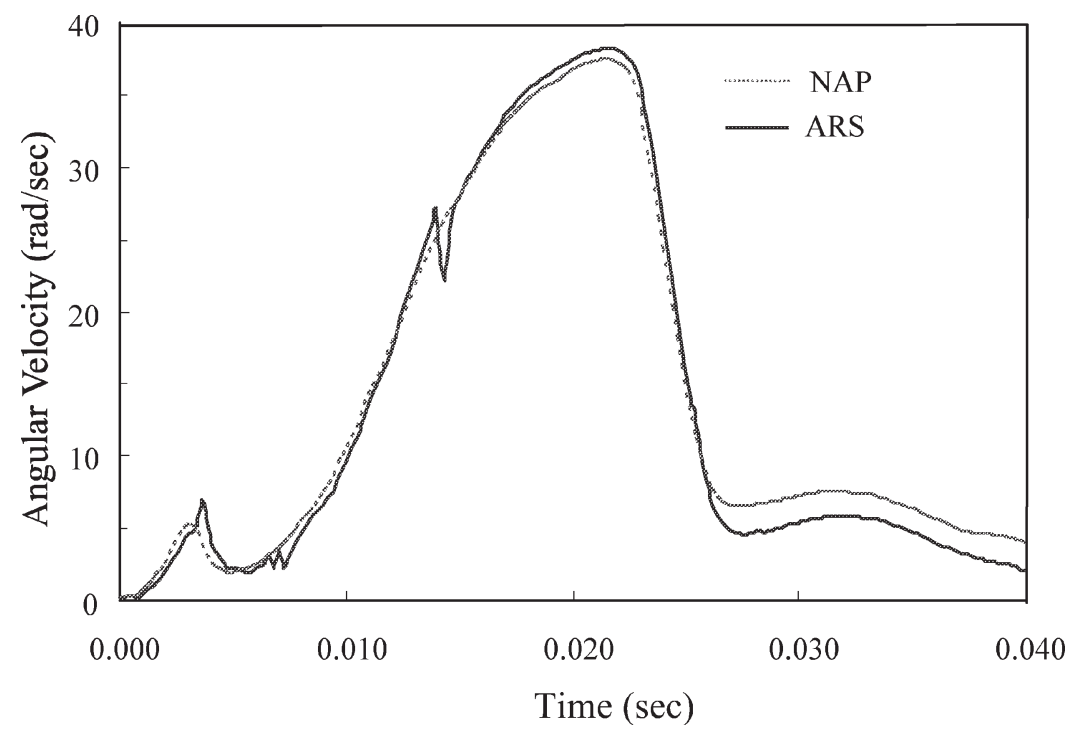

Fig. 11b. Contact test, resultant angular velocity. 
via the periphery scheme is expressed by combining Eqs (8) and (22):

$$
\begin{gathered}
\left(\Delta A_{0 X}\right)^{2}=\left(\Delta A_{1 X}\right)^{2}(1)^{2}+\left(\Delta \omega_{Y}\right)^{2}\left(2 \omega_{Y} r_{X}\right)^{2} \\
+\left(\Delta \omega_{Z}\right)^{2}\left(2 \omega_{Z} r_{X}\right)^{2}+\left(\Delta r_{X}\right)^{2}\left(\omega_{Y}^{2}+\omega_{Z}^{2}\right)^{2}
\end{gathered}
$$

In a similar manner, the error expressions for the other components of linear and angular acceleration may be written for the periphery scheme (using Eqs (9)-(13)) and the compact cube schemes (using Eqs (14)-(16)). The maximal errors in the angular acceleration terms for the compact cube scheme are simply the $1.5 \%$ of the value computed by differentiating the corresponding angular velocity terms measured by the ARS.

The error sensitivity due to position uncertainties may be examined for the computations involving head form impact tests. Rather than examining the entire time-histories of linear acceleration and angular acceleration, error sensitivity limits are computed at three discrete times of particular interest: the times when the magnitudes of the angular acceleration, the angular velocity, and the acceleration at the head form CG are highest. Conceptual values of these terms are given in Table 3 for a hypothetical contact test with motion in a single plane. The values correspond closely to those seen in the actual contact test (Fig. 6).

The resultant of the acceleration vector at the head form's CG, the angular acceleration of the head form, and the associated error of each are given for each scheme in Table 4. Depending on the computed quantity desired, the precision of sensor position measurements may or may not be adequate. If peak linear acceleration values are desired, then the error limits of sensor positions are sufficient. If linear acceleration in the presence of peak angular velocity is desired, then more precise position measurements are needed.

The error analysis can also help decide which position measurements - distance (vector $\vec{r}$ ), orientation, or both - need to be improved. For example, when the magnitude of the linear acceleration vector is highest, misalignments of the triax blocks are responsible for almost all of the overall error. Conversely, errors in the distance measurements contribute most significantly when the angular velocity is greatest.

The sources of error considered in this analysis are sensor orientation and the radial distance from the body-fixed origin. Errors from theses sources may be reduced by improving test procedures. Other error sources are also present, but they are not included in this analysis because they cannot be controlled by a test technician as easily. These include errors due to signal noise, cross-axis sensitivity, calibration, and sensor misreadings, and noise magnification during the numerical differentiation of ARS data.

\section{Conclusions}

Three sensor schemes - the NAP, the periphery, and the compact cube - designed to measure the sixdegree-of-freedom acceleration of a rigid body were compared in $4.8 \mathrm{~m} / \mathrm{s}$ impact tests using a $3.6 \mathrm{~kg}$ aluminum head form. The resulting acceleration timehistories covered about $50 \mathrm{~ms}$. From a signal processing standpoint, all three schemes were highly correlated near the time of peak acceleration. The differences in values were likely due to errors in sensor positioning and sensitivity factors. However, under certain circumstances, one scheme may be preferable to another.

In noisy environments that give rise to unwanted surface vibrations on the rigid body, the periphery scheme provides the best results. The quality of the data produced by the compact cube scheme (which uses numerically differentiated ARS data) is highly influenced by the slightest amount of signal noise. Even in a relatively noise-free environment, ARS signals require filtering prior to numerical differentiation.

Noise also affects the NAP scheme more than the periphery scheme. The NAP scheme relies entirely upon data from accelerometers which are more sensitive to vibrations than the ARS sensors used in the periphery scheme. The NAP is best suited to hollow rigid bodies with built-in sensor mounting locations like those in the test head form. Although a NAP mounting fixture could be used for exterior applications, it would probably be even more susceptible to surface vibrations. Also, the NAP relies on integrated data to compute linear acceleration in such a setup, so error accumulation could be a problem.

For both of the head form tests, a post-test digital low-pass filter was imposed on the ARS signals ( $300 \mathrm{~Hz}$ cut-off frequency) and the accelerometer signals (600 Hz cut-off frequency). The drawback of filtering data is that high frequency, non-vibrational rigid body movements may be lost. Of the two impact tests performed, the contact test produced the higher nonvibrational frequencies. Despite the digital filters, the peak accelerations were still captured, indicating there 


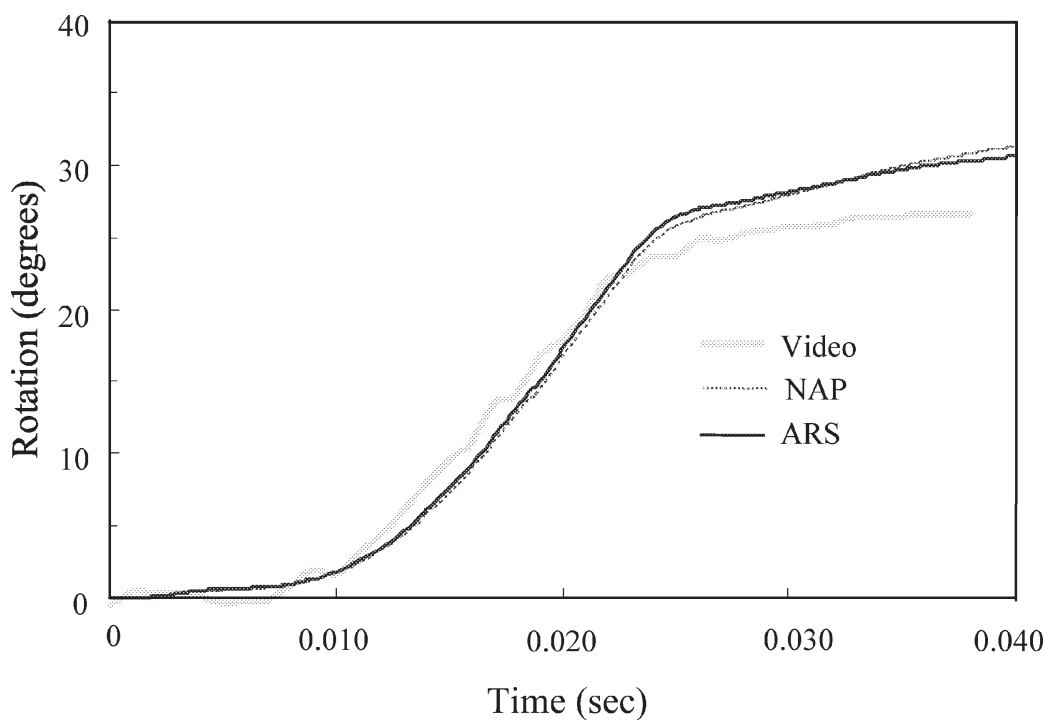

Fig. 12. Contact test, Y-axis rotation.

Table 3

Typical values for the contact test shown in Fig. 5

\begin{tabular}{|c|c|c|c|c|}
\hline \multirow[t]{3}{*}{ Sensor (see Fig. 4) } & \multicolumn{4}{|c|}{ Sensor reading @ timepoint (coincides with max./min.) } \\
\hline & t1 @ & t2 @ & t3 @ & Maximal \\
\hline & $\left|\vec{A}_{C G}\right|(\max )=263 \mathrm{~g}$ & $\left|\dot{\omega}_{Y}\right|(\max )=10,000 \mathrm{rad} / \mathrm{s}^{2}$ & $\left|\vec{\omega}_{Y}\right|(\max )=40 \mathrm{rad} / \mathrm{s}$ & error, $\Delta$ \\
\hline Compact cube $A_{X}(\mathrm{~g})$ & -240 & -45 & 15 & $\pm 1.5 \%$ \\
\hline Compact cube $A_{Z}(\mathrm{~g})$ & -100 & 80 & -10 & $\pm 1.5 \%$ \\
\hline Peripheral $A_{X}(\mathrm{~g})$ & 240 & -45 & 15 & $\pm 1.5 \%$ \\
\hline Peripheral $A_{Z}(\mathrm{~g})$ & -108 & 5.5 & -31.9 & $\pm 1.5 \%$ \\
\hline Angular velocity, $\omega_{Y}(\mathrm{rad} / \mathrm{s})$ & -5 & -23 & -40 & $\pm 1.5 \%$ \\
\hline Angular acceleration, $\dot{\omega}_{Y}\left(\mathrm{rad} / \mathrm{s}^{2}\right)$ & 900 & 10,000 & 80 & $\pm 1.5 \%$ \\
\hline Distance, $r_{X}(\mathrm{~m})$ & -0.08 & -0.08 & -0.08 & $\pm 5.0 \%$ \\
\hline Distance, $r_{Y}(\mathrm{~m})$ & -0.10 & -0.10 & -0.10 & $\pm 5.0 \%$ \\
\hline Distance, $r_{Z}(\mathrm{~m})$ & 0.13 & 0.13 & 0.13 & $\pm 5.0 \%$ \\
\hline
\end{tabular}

Table 4

Error sensitivity in the periphery and compact cube schemes

\begin{tabular}{lcccc}
\hline Timepoint: & Computed & Computed & \multicolumn{2}{c}{ Computation scheme (error, \%) } \\
(coincides with max./min.) & term & value & Peripheral & Compact cube \\
\hline t1 @ $\left|\vec{A}_{C G}\right|(\max )=263 \mathrm{~g}$ & $\left|\vec{A}_{C G}\right|$ & $263 \mathrm{~g}$ & $\pm 1.50 \%$ & $\pm 1.50 \%$ \\
& $\dot{\omega}_{Y}$ & $900 \mathrm{rad} / \mathrm{s}^{2}$ & $\pm 7.30 \%$ & $\pm 1.50 \%$ \\
& $\left|\vec{A}_{C G}\right|$ & $10.8 \mathrm{~g}$ & $\pm 1.70 \%$ & $\pm 2.87 \%$ \\
$\mathrm{t} 2 @ \omega_{Y}(\min )=-40 \mathrm{rad} / \mathrm{s}$ & $\dot{\omega}_{Y}$ & $80 \mathrm{rad} / \mathrm{s}^{2}$ & $\pm 0.93 \%$ & $\pm 1.50 \%$ \\
& $\left|\vec{A}_{C G}\right|$ & $49.3 \mathrm{~g}$ & $\pm 14.3 \%$ & $\pm 9.59 \%$ \\
$\mathrm{t} 3 @ \dot{\omega}_{Y}(\max )=10,000 \mathrm{rad} / \mathrm{s}^{2}$ & $\dot{\omega}_{Y}$ & $10,000 \mathrm{rad} / \mathrm{s}^{2}$ & $\pm 24.7 \%$ & $\pm 1.50 \%$ \\
& & & & \\
\hline
\end{tabular}


was very little head motion at frequencies higher than the filter cut-offs. This may not have been the case had the impact velocity been higher and the sorbethane pad thinner.

If angular velocity or rotation is a concern, the ARSbased periphery and compact cube schemes are preferable to the NAP. ARS sensors measure angular velocity directly, while the NAP scheme numerically integrates acceleration data once to obtain angular velocity, and twice to obtain rotation. Although there is almost no difference between the NAP and the ARS data in Figs 10-11, error accumulations in the integrated NAP data would probably appear if the time-histories were longer.

From a physical point of view, the compact cube scheme is easiest to use and is the most economical since it requires just six sensors. (The unit cost of the ARS sensors used in the test series was about the same as the unit accelerometer cost.) Although the periphery scheme needs just six sensors to measure the linear acceleration of an inaccessible point, it needs three more to find angular acceleration. The NAP scheme also requires nine sensors.

The compact cube scheme also has the smallest configuration $(85 \mathrm{~g})$ and it may be mounted anywhere on the rigid body. The periphery scheme is the most difficult system to implement physically. It requires three mounting sites and cannot be used to measure the acceleration of inaccessible points that lie on the surface of a rigid body. However, the periphery scheme could be adapted to a co-planar scheme that could be arranged on a flat mounting plate and fastened to the rigid body at any site. The NAP operates best when mounted within a spherical and hollow rigid body. For a solid, rigid body, the NAP must be used in conjunction with a relatively large (200 g) mounting fixture that may affect the inertial properties of the body.

\section{References}

[1] F.P. DiMasi, Transformation of Nine-Accelerometer-Package (NAP) data for replicating headpart kinematics and dynamic loading. NHTSA Technical Report, DOT HS 808 282, National Technical Information Service, Springfield, VA, 1996.

[2] E.O. Doebelin, Measurement Systems Application and Design, McGraw-Hill, New York, 1990.

[3] D.R. Laughlin, A magnetohydrodynamic angular motion sensor for anthropomorphic test device instrumentation. Society of Automotive Engineers, Warrenton, PA, Paper No. 892428 (1989).

[4] Y.K. Liu, Discussion of "Measurement of angular acceleration of a rigid body using linear accelerometers", J. Appl. Mechanics 43 (1976), 377-378.

[5] A.J. Padgaonkar, K.W. Krieger and A.I. King, Measurement of angular acceleration of a rigid body using linear accelerometers, J. Appl. Mechanics 42 (1975), 552-556.

[6] G. Plank, H. Weinstock, M. Coltman and H. Lee, Methodology for the calibration of and data acquisition with a six-degree-offreedom acceleration measurement system. NHTSA Technical Report, DOT HS 807-370, National Technical Information Service, Springfield, VA, 1989.

[7] SAE Information Report, Human tolerance to impact conditions as related to motor vehicle design. Society of Automotive Engineers, Warrenton, PA, SAE J885 (1986).

[8] SAE Recommended practice, Instrumentation for impact test, I. Electronic instrumentation. Society of Automotive Engineers, Warrenton, PA, SAE J211 (1995).

[9] D.C. Viano, J.W. Melvin, J.D. McCleary, R.G. Madeira, T.R. Shee and J.D. Horsch, Measurement of head dynamics and facial contact forces in the Hybrid III dummy. Society of Automotive Engineers, Warrenton, PA, Paper No. 861891 (1986). 

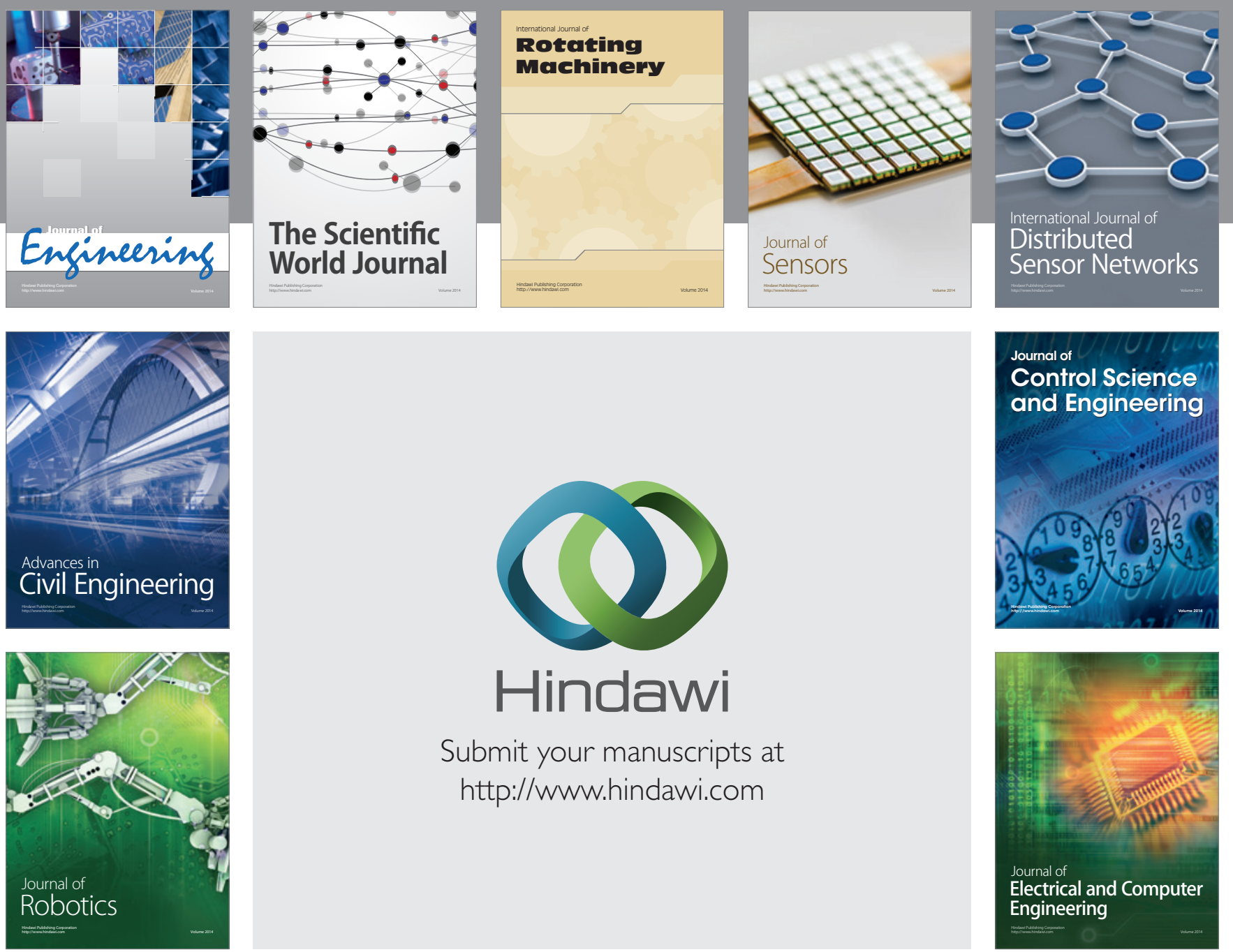

Submit your manuscripts at

http://www.hindawi.com
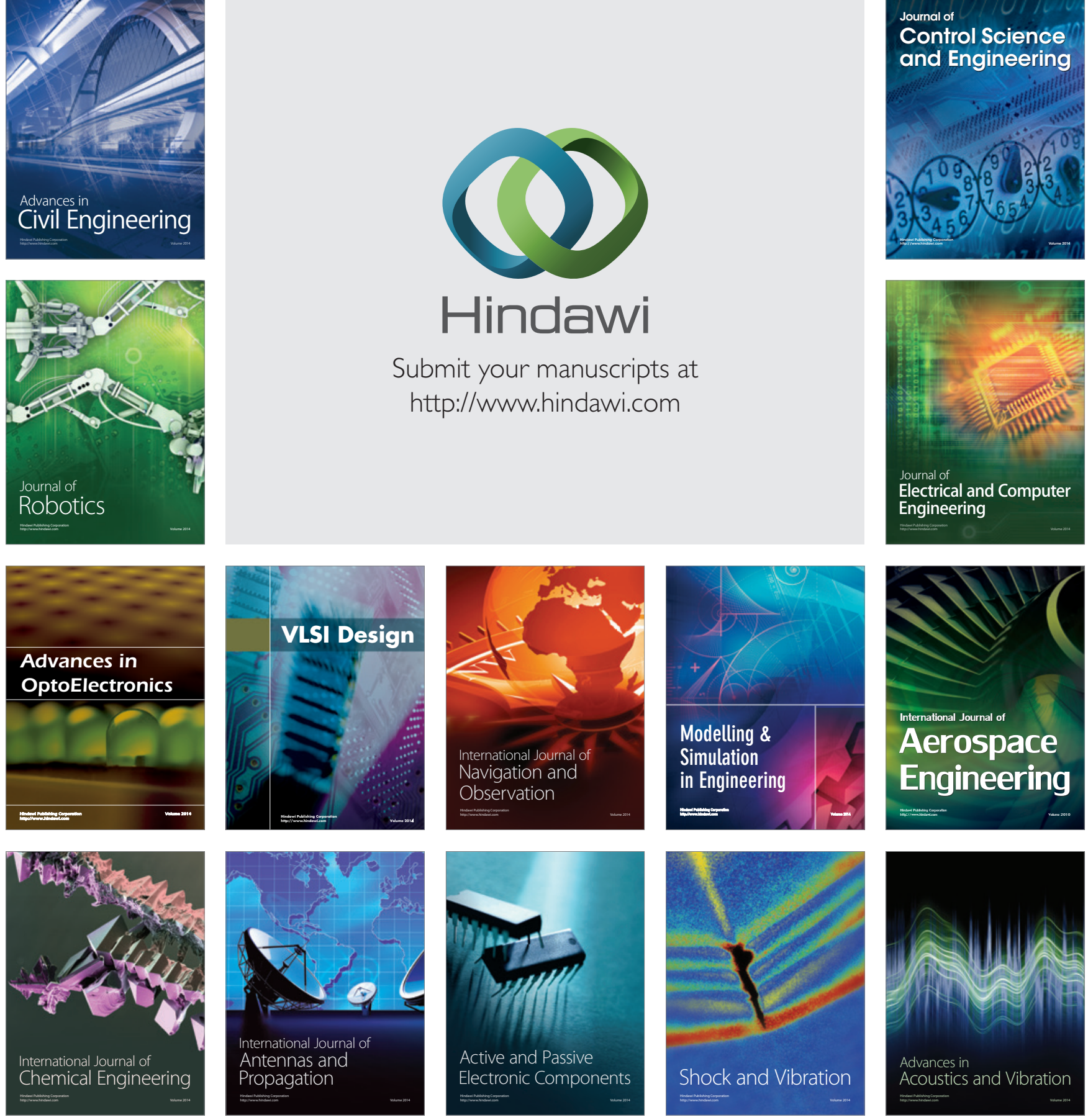